\title{
Factors Associated with Physical-Activity Performance by Older Individuals in a Medium-Sized City in São Paulo State, Brazil
}

\author{
José Eduardo Corrente ${ }^{1}$, Giovana Fumes ${ }^{2}$ and Tania Ruiz ${ }^{3}$ \\ 1. Biostatistics Department, Biosciences Institute of Botucatu, UNESP, São Paulo18618-970, Brazil \\ 2. MsC in Biometry, Biostatistics Department, Biosciences Institute of Botucatu, UNESP, Botucatu, São Paulo18618-970, Brazil \\ 3. Public Health Department, Botucatu School of Medicine, UNESP, Botucatu, São Paulo18618-970, Brazil
}

Received: September 18, 2012 / Accepted: November 14, 2012 / Published: February 28, 2013.

\begin{abstract}
Physical activity has been scientifically discussed as fundamental in the process of healthy ageing. Hence, this study aimed at determining the factors that influence older people to perform physical activities. The complete IPAQ (International Physical Activity Questionnaire) was applied to a population-based sample consisting of 364 elderly persons in the city of Botucatu, São Paulo, Brazil. Days of physical activity performed by the older people were considered by taking into account household and leisure activities. Models for count data were fitted by including socio-demographic variables as well as those related to life satisfaction. It was shown that housework physical-activity performance is associated with female, who predominantly showed to be more active in all levels. Male seemed to be more predisposed to perform lighter recreation, sports and leisure-time physical activities, such as walking. Additionally, poor schooling showed to be decisive for not performing physical activities both at home and during leisure.
\end{abstract}

Key words: Older people, physical activity, models for count data.

\section{Introduction}

According to the WHO (World Health Organization), health implies a state of complete physical, mental and social. So being healthy is not simply the absence of disease, but there is a concern about the healthy life expectancy and quality. In this aspect, physical activity has been increasingly discussed and analyzed scientifically as fundamental aging process and its benefits of healthy active lifestyle, quality of life, control of chronic diseases, mortality and maintaining capacity functional during this process [1-5]. Physical activity is defined as bodily movement produced by skeletal muscle action that results in energy expenditure above resting [6]. Monitoring levels of physical activity in

Corresponding author: José Eduardo Corrente, associate Professor, research field: epidemiology of third age. Email: jecorren@ibb.unesp.br the elderly has become a subject of interest and concern among experts. The most frequently used to assess physical activity are the questionnaires, among which stands out the IPAQ (International Physical Activity Questionnaire) [7] which has been validated in Brazil for older men and women [8-9].

IPAQ investigates the number of days and the time spent in daily physical activities per week, which are measured in the following domains: work-related, transportation, housework/gardening and leisure-time. Three levels of physical activity are considered light, moderate and vigorous, and the results of the questionnaire application classify individuals as sedentary, active and very active by taking into account the time spent in physical activity in each of the domains. 
Since the time periods spent in physical activity are always reported and such values may not reflect reality, particularly with regard to older individuals, thus can lead to a misclassification level for the activities. Also, depending on the classification of the physical activity performed by older people, the factors associated with such practice are not usually found.

As IPAQ also investigates the number of days of physical activities, another form to evaluate the determinants of the practice or non-practice may be these frequencies, disregarding the reported daily time for the activity performed [10]. In this case, it is not possible to establish a classification level of the activities, but only the practice or non-practice. It was not found in literature articles that analyzes only the frequency of physical activities for older individuals as well as related factors with the practice or non-practice.

Therefore, this study aimed at investigating the factors that are associated with the practice or not of physical activities by older individuals based on the number of days per week as assessed by IPAQ in a medium-sized city in São Paulo state, Brazil.

\section{Methodology}

This is a first study using real data from IPAQ that analyzes the frequencies of physical activities in older individuals. For that, a population inquiry was performed to study the profile, style and quality of life of the population aged 60 years and older by using a multidimensional instrument in the city of Botucatu (120 thousand inhabitants), São Paulo, Brazil, in 2003.

The sample planning consisted of a systematic sample of households from a database containing nine thousand families randomly allocated during the conduction of a study on labor accidents in the city in 2000 [11]. From that database, the residents aged 60 years and older were randomly identified.

The sample size $(n=384)$ was obtained by taking into account a sample error of $0.05 ; z$ value equal to 1.96, relative to a confidence interval of $95 \%$ (two-sided $\alpha$ of 0.025 ), and the prevalence of the characteristic of interest equal to 0.5 . There was a $5 \%$ loss from the initially estimated sample, totaling 364 older individuals.

The completed IPAQ and questions concerning socio-demographic conditions, habits and lifestyle, reported morbidity, antecedents and quality of life were the instruments used for data collection. The number of days obtained on IPAQ for daily physical activity performed at home and in leisure and socio-demographic variables were used for analysis. The selection of domains, physical activity at home and during leisure, was characterized by the fact that the sample showed more than $80 \%$ of older individuals who reported not to work anymore.

By considering the response variable as the number of days of activity in these domains, the Poisson and Negative Binomial models for count data were fitted, and, due to the large number of zeros present in the data, the Zero-Inflated Poisson and Zero-Inflated Negative Binomial models were also tested in function of the socio-demographic variables. Graphs are shown in order to evaluate the quality of fitting of such models [12]. The use of inflated and non-inflated models was made through the Wuong test [13]. The fitting between the inflated models was verified according to the Akaike Information Criterion-AIC and the Bayes Information Criterion-BIC [14].

The whole research process followed the ethical principles provided for in Resolution No. 196/96 by the National Health Council, Ministry of Health, which ensures to participants, among other rights, their free consent provision as well as confidentiality and non-disclosure of information. The research project was approved by the Research Ethics Committee of the Botucatu School of Medicine of São Paulo State University (UNESP) on Nov. 11, 2002. 


\section{Results and Discussion}

\subsection{Characterization of the Sample and the} Frequencies of Physical activities

The initial data analysis showed that the older individuals' mean age was 74.2 (S.D. $=7.3$ ) years and that their mean time of residence in the city was 47.2 $(S . D .=21.6)$ years. Table 1 shows a descriptive analysis of the older residents' socio-demographic data in the city of Botucatu.

As observed in Table 1, of the 364 older individuals interviewed, 58.5\% $(n=213)$ were females, 56.8\% ( $n$ = 206) were married, $72.3 \%(n=263)$ had graduated from elementary school, $80.2 \%(n=292)$ were reported not to work anymore, $65.9 \%(n=240)$ were retired, and 95.6\% $(n=347)$ declared to be satisfied about life.

According to IPAQ, physical activities at home were considered according to the intensity below:

- Light-carrying light weights, cleaning glass windows or doors, sweeping and cleaning the floor, doing the dishes, dusting inside the house for at least 10 continuous minutes;

- Moderate-carrying light weights, cleaning glass windows or doors, raking and sweeping outside the house for at least 10 continuous minutes;

- Vigorous-hoeing, washing the back yard, scrubbing the floor and washing clothes for at least 10 continuous minutes.

Also, the recreation, sports exercise and leisure-time, the intensity were:

- Light-walking for at least 10 continuous minutes;

- Moderate-pedaling or swimming at regular speed, playing ball, volleyball, basketball, tennis for at least 10 continuous minutes;

- Vigorous-aerobic exercises, swimming or pedaling fast, running for at least 10 continuous minutes.

Table 2 shows the distribution of the number of days of physical activity performed by the older individuals in relation to household chores, family care provision and recreation, sports and leisure activities.

As shown in Table 2, for domain physical activity at home, it was observed that a large percentage of the older individuals performed light activities on the seven days of the week $(45.9 \%, n=167)$. In contrast, large percentages of individuals who did not perform moderate $(71.4 \%, n=260)$ or vigorous $(69.2 \%, n=$ 252) activity were observed for that domain as well as

Table 1 Distribution of older individuals according to socio-demographic variables. Botucatu, 2003.

\begin{tabular}{llc}
\hline Variables & Categories & $n(\%)$ \\
\hline Gender & Male & $151(41.48)$ \\
\multirow{3}{*}{ Marital status } & Female & $213(58.52)$ \\
& Total & $364(100.00)$ \\
Education & Unmarried & $157(43.25)$ \\
& Tarried & $206(56.75)$ \\
Still working & Elementary school education & $363(100.00)$ \\
& Higher than elementary school education & $263(72.25)$ \\
Retired & Total & $101(27.75)$ \\
& Yes & $364(100.00)$ \\
Satisfied about life & No & $72(19.78)$ \\
& Total & $292(80.22)$ \\
& Yes & $364(100.00)$ \\
& No & $240(65.93)$ \\
& Total & $124(34.07)$ \\
& Yes & $364(100.00)$ \\
& No & $347(95.59)$ \\
& Total & $16(4.41)$ \\
\end{tabular}


in relation to recreation, sports and leisure, in which most of the individuals did not perform light $(58.2 \%$, $n=212)$, moderate $(93.1 \%, n=339)$ or vigorous $(95.1 \%, n=346)$ activities on any days of the week. By considering that sedentariness refers to not practicing any type of physical activity at least once a week, it was observed that the older residents of Botucatu showed a high prevalence of sedentariness [15].

\subsection{Zero-inflated Models Fitted for Housework} Domain

Table 3 shows the models fitted for each level of physical activity obtained by IPAQ in the housework domain.

For domain housework physical activity in the vigorous level, the model that best fitted the data was the ZINB (zero-inflated negative binomial model), as

Table 2 Frequency and percent of older individuals referring to the number of days of physical activity related to household chores and recreation. Botucatu, 2003.

\begin{tabular}{lccccccc}
\hline & \multicolumn{2}{c}{ Physical activity at home - household chores and family } & & \multicolumn{2}{c}{ Recreation, sports, exercise and leisure - time physical } \\
Noctivity
\end{tabular}

Table 3 Fitting of the zero-inflated negative binomial model for the housework activity domain (vigorous and intense levels) and fitting of the zero-inflated poisson model (light level) in older individuals, 2003.

\begin{tabular}{|c|c|c|c|c|c|c|}
\hline Levels & Variables & Categories & Estimate & S.E. & $P$ value & OR (CI95\%) \\
\hline \multicolumn{7}{|l|}{ Vigorous } \\
\hline \multirow{6}{*}{ Inflated part } & Age & & 0.066 & 0.021 & 0.002 & $1.068(1.025 ; 1.112)$ \\
\hline & Gender & Male & 0.951 & 0.287 & 0.001 & 2.587 (1.475; 4.538) \\
\hline & & Female & - & - & - & 1.000 \\
\hline & Schooling & Elementary school & -0.699 & 0.320 & 0.029 & 0.497 (0.265; 0.931) \\
\hline & & $\begin{array}{l}\text { Higher than } \\
\text { elementary school }\end{array}$ & - & - & - & 1.000 \\
\hline & & Wuong Test & $z=3.300$ & $p=0.005$ & $\mathrm{AIC}=878.265$ & $\mathrm{BIC}=901.632$ \\
\hline \multicolumn{7}{|l|}{ Moderate } \\
\hline \multirow{5}{*}{ Inflated part } & Gender & Male & 0.719 & 0.287 & 0.012 & 2.053 (1.169; 3.605) \\
\hline & Gender & Female & - & - & - & 1.000 \\
\hline & Still working & No & 0.773 & 0.343 & 0.024 & 2.167 (1.107; 4.242) \\
\hline & & Yes & - & - & - & 1.000 \\
\hline & & Wuong Test & $z=2.350$ & $p=0.009$ & $\mathrm{AIC}=895.585$ & $\mathrm{BIC}=915.071$ \\
\hline \multicolumn{7}{|l|}{ Light } \\
\hline \multirow[t]{2}{*}{ Inflated part } & Satisfaction & Not Satisfied & 1.081 & 0.520 & 0.038 & 2.948 (1.065; 8.166) \\
\hline & about life & Satisfied & - & - & - & 1.000 \\
\hline \multirow{4}{*}{ Non-inflated part } & & & & & & RP (CI95\%) \\
\hline & & Male & -0.231 & 0.062 & 0.000 & $0.794(0.703 ; 0.897)$ \\
\hline & Gender & Female & - & - & - & 1.000 \\
\hline & & Wuong Test & $z=8.830$ & $P=0.000$ & $\mathrm{AIC}=1,532.731$ & $\mathrm{BIC}=1,548.309$ \\
\hline
\end{tabular}


shown in Table 3. By fitting only the zero-inflated part in this model, significant associations were obtained for elderly individuals at older ages (OR = 1.068 ; $\mathrm{CI} 95 \%=1.025 ; 1.112)$ who were males $(\mathrm{OR}=$ 2.587; CI95\% = 1.475; 4.538) and had poor education $(\mathrm{OR}=0.497 ; \mathrm{CI} 95 \%=0.265 ; 0.931)$. Hence, poorly educated male elderly individuals at older ages were less likely to perform vigorous physical activity at home. As to the moderate level, following the same model, significant associations occurred again with male older individuals (OR $=2.053$; CI95\% $=1.169$; $3.605)$ who did not work $(\mathrm{OR}=2.167$; CI95\% $=1.107$; 4.242). Hence, male older individuals who did not work were less likely to perform moderate physical activity while doing housework.

For the light level, a zero-inflated Poisson model was fitted. Male older individuals $(\mathrm{OR}=0.794$; CI95\% $=0.703 ; 0.897$ ) showed a protection factor to the performance of easier household chores. The greater likelihood of non-performance at this level was associated with older individuals who were not satisfied about life $(\mathrm{OR}=2.948$; CI95\% $=1.065$; 8.166).

In general, housework physical activities showed to be less likely to be performed by male older individuals in all levels studied. As to the vigorous level, the older an individual, the greater the probability of his not performing this type of activity due to the very limitations imposed by nature. The low-schooling factor appeared in the same level, revealing that older individuals with poorer schooling tended to not perform heavier tasks at home. As to the moderate level, older individuals who reported not to work anymore showed a greater probability of not performing activities at this level, being more inactive than those who were still working. As regards light activities, older individuals who were not satisfied about life showed greater probability of not performing simple household chores, which was possibly due to the lack of enthusiasm in relation to their own lives.

In order to evaluate whether the fitting of models in Table 3 was adequate, Figs 1,2 and 3 were generated. Such figures show the difference between the observed proportion of zeros and the possibilities predicted by the count models. From the figures, it is observed that the difference between the sampled proportion of zeros and the probabilities estimated for the zero-inflated models (ZIP and ZINB) was close to zero, thus confirming the good fitting of modeling, particularly with regard to the non-performance of physical activity. In Fig 3, the

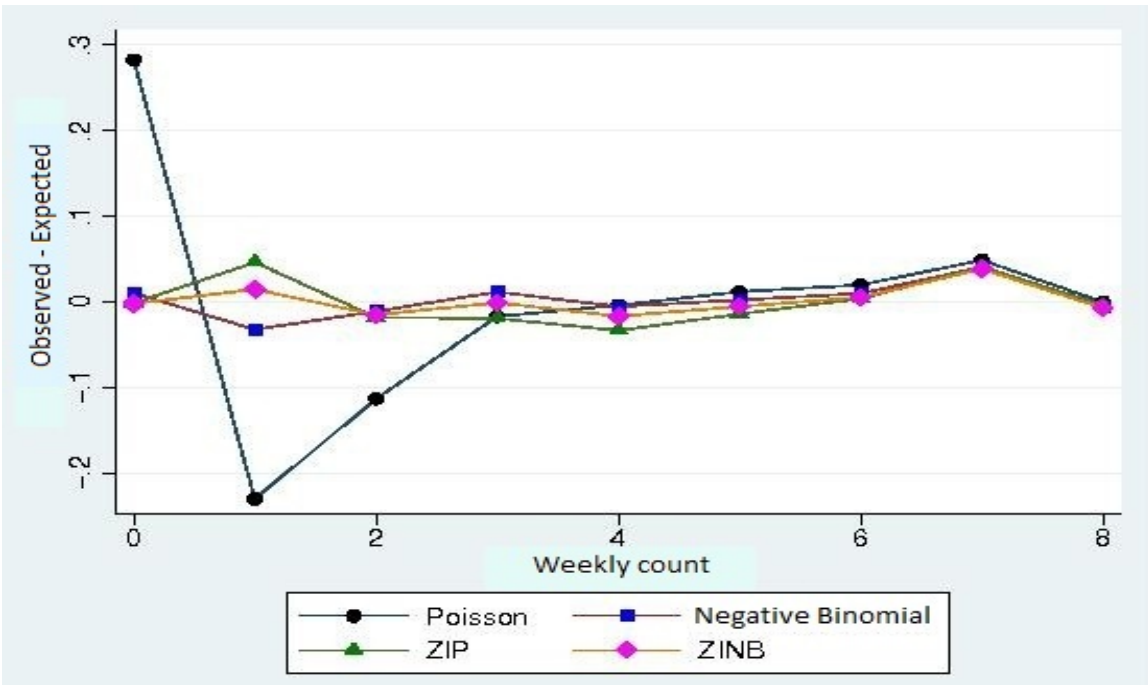

Fig. 1 Difference between the proportion observed and the mean probabilities from the four models fitted for housework physical-activity performance (rigorous level). Botucatu, 2003. 


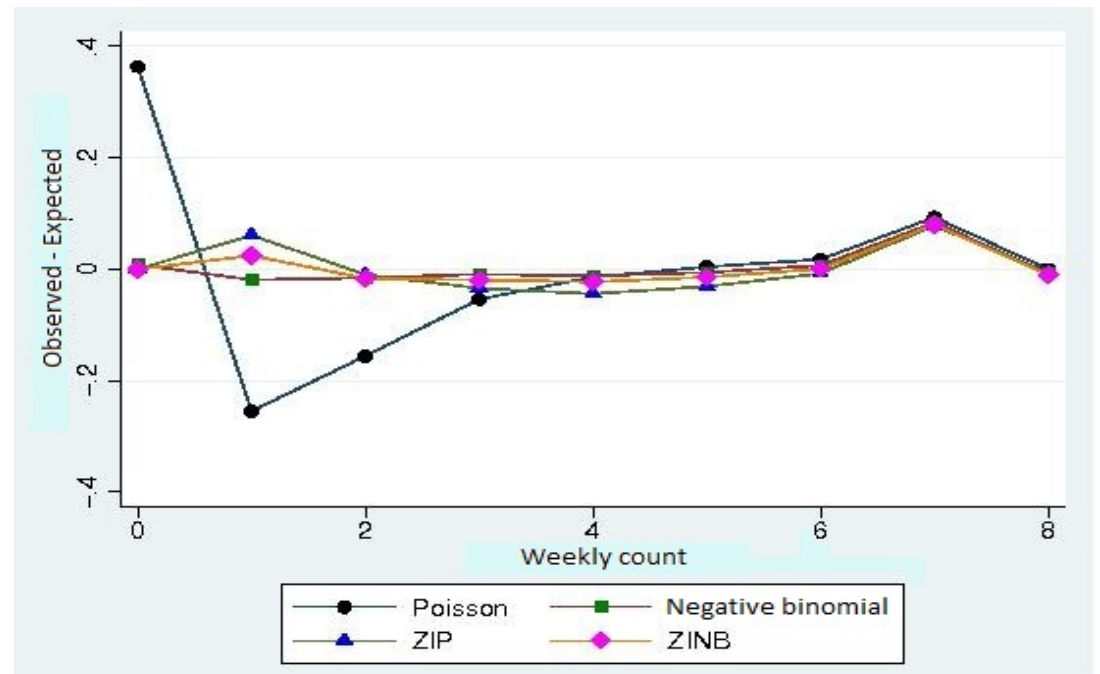

Fig. 2 Difference between the proportion observed and the mean probabilities from the four models fitted for housework physical-activity performance (moderate level). Botucatu, 2003.

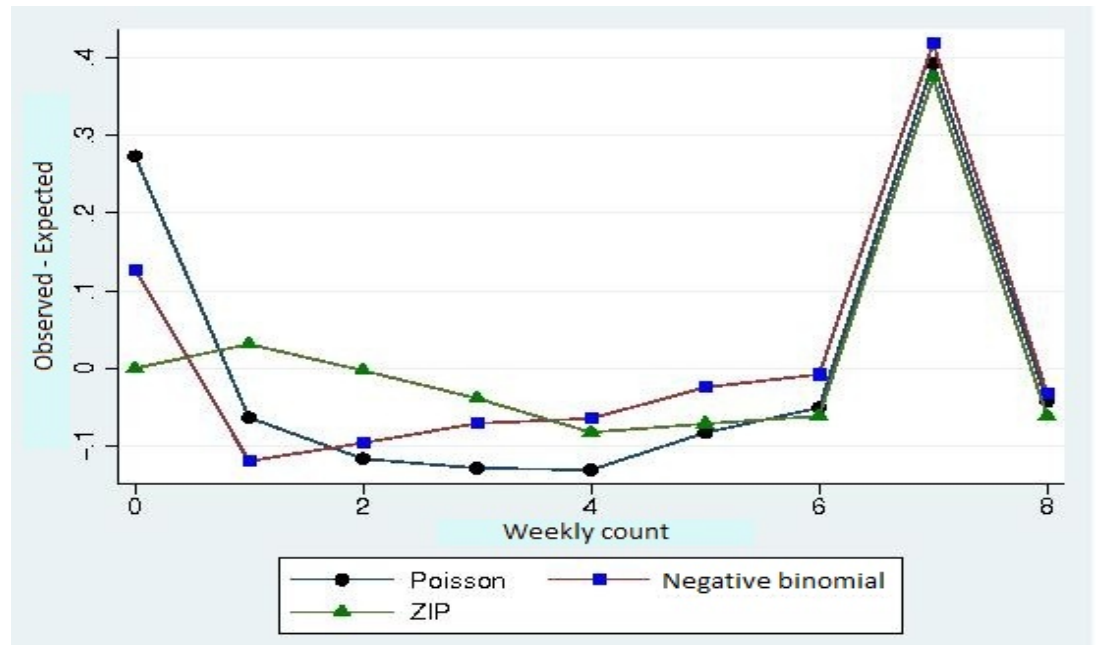

Fig. 3 Difference between the proportion observed and the mean probabilities from the four models fitted for housework physical-activity performance (light level). Botucatu, 2003.

convergence of the interactive process used in the ZINB model for the fitting with significant covariates is noteworthy.

\subsection{Zero-inflated Models Fitted for Leisure-time}

Table 4 shows the models fitted for each physical activity level obtained by IPAQ in the domain leisure-time physical activity.

Considering the domain eisure-time physical activity, the best model fitted was the Zero-Inflated Poisson model (ZIP) for all levels. As shown in Table 4, significant associations were obtained in the inflated part for the rigorous and moderate levels, with unmarried older individuals (rigorous: OR = 6.522; CI95\% $=1.468 ; 28.967$; moderate: OR = 3.266; CI95\% = 1.188; 8.974 ) and individuals with poorer schooling (intense: OR $=2.743$; $\mathrm{CI} 95 \%=$ 1.038; 7.246; moderate: $\mathrm{OR}=2.579 ;$ CI95\% = 1.121; 5.937), who showed greater likelihood to not perform physical activities in these levels for the leisure-time domain. Concerning walking as physical activity (considered to be light level), the only factor associated was gender. Male older individuals showed smaller probability of not practicing such activity (OR $=0.599 ; \mathrm{CI} 95 \%=0.391 ; 0.918)$ in relation to women. 
Table 4 Fitting of the Zero-Inflated Poisson Model for domain leisure-time physical activity (vigorous, moderate and light levels) in older individuals. Botucatu, 2003.

\begin{tabular}{|c|c|c|c|c|c|c|}
\hline Levels & Variables & Categories & Estimate & S.E. & $P$ value & OR (CI95\%) \\
\hline \multicolumn{7}{|l|}{ Rigorous } \\
\hline & Marital status & Unmarried & 1.875 & 0.761 & 0.014 & 6.522 (1.468; 28.967) \\
\hline \multirow[t]{4}{*}{ Inflated part } & & Married & - & - & - & 1.000 \\
\hline & Schooling & Elementary school & 1.009 & 0.496 & 0.042 & 2.743 (1.038; 7.246) \\
\hline & & $\begin{array}{l}\text { Higher than elementary } \\
\text { school }\end{array}$ & - & - & - & 1.000 \\
\hline & & Wuong Test & $z=3.920$ & $p(z)=0.000$ & AIC $=206.725$ & $\mathrm{BIC}=222.302$ \\
\hline \multicolumn{7}{|l|}{ Moderate } \\
\hline & Marital status & Unmarried & 1.183 & 0.516 & 0.022 & $3.266(1.188 ; 8.974)$ \\
\hline \multirow[t]{4}{*}{ Inflated part } & & Married & - & - & - & 1.000 \\
\hline & Schooling & Elementary school & 0.948 & 0.425 & 0.026 & $2.579(1.121 ; 5.937)$ \\
\hline & & $\begin{array}{l}\text { Higher than elementary } \\
\text { school }\end{array}$ & - & - & - & 1.000 \\
\hline & & Wuong test & $\mathrm{z}=4.450$ & $p(z)=0.000$ & AIC $=278.684$ & $\mathrm{BIC}=294.261$ \\
\hline \multicolumn{7}{|l|}{ Light } \\
\hline \multirow[t]{3}{*}{ Inflated part } & Gender & Male & -0.512 & 0.019 & 0.019 & $0.599(0.391 ; 0.918)$ \\
\hline & & Female & - & - & - & 1.000 \\
\hline & & Wuong Test & $z=15.340$ & $p(z)=0.000$ & AIC $=1,165.219$ & $\mathrm{BIC}=1,176.911$ \\
\hline
\end{tabular}

Hence, unmarried older individuals are not stimulated to practice physical activities, as it was found for rigorous and moderate levels. Also, in these levels, poor schooling appeared as a limiting factor to activity performance at least in predominantly urban areas, such as in this study. Some other investigations report that leisure-time physical activity is positively related to the socio-economic level adopted. Individuals with poor schooling levels tend to more frequently adopt life habits that do not preserve life because they have less access to information and their life conditions do not probably enable the incorporation of healthy behaviors [16]. Male older individuals seem to be more prone to go for walks.

The older individuals in this study reflect a situation of sedentariness that is common in Brazil. Studies show that $58 \%$ of the older people in the country's southern and northern regions do not perform physical activity [17]. Another study conducted in the northern and southern regions shows that only $8.7 \%$ of men and $6.8 \%$ of women aged 60 years or over reported to perform some type of physical activity, which is regarded as such when practiced for at least thirty minutes once a week [18]. This result agrees with the findings, since $8.5 \%$ of the men and $7.4 \%$ of the women in the study performed leisure activities. Similar results in which the full magnitude of physical activity are more prevalent among men during leisure activities [16].

Again, in order to evaluate the fitting of models, Figs. 4-6 show a comparison of the four count models used in modeling, pointing out the good fitting of the zero-inflated models (ZIP and ZINB), particularly as regards the non-performance of leisure-time physical activity. In Fig. 6, the non-convergence of the iteractive process used in fitting the ZINB model associated with significant covariates is noteworthy.

\section{Conclusion}

This study provided an integrated characterization of the factors that influence the performance or not of housework and leisure-time physical activity. As a result, it was shown that housework physical-activity performance is associated with female older individuals, who predominantly showed to be more active in all levels. In turn, male older individuals seemed to be more predisposed to perform lighter recreation, sports and leisure-time physical activities, 
Factors Associated with Physical-Activity Performance by Older Individuals in a Medium-Sized City in São Paulo State, Brazil

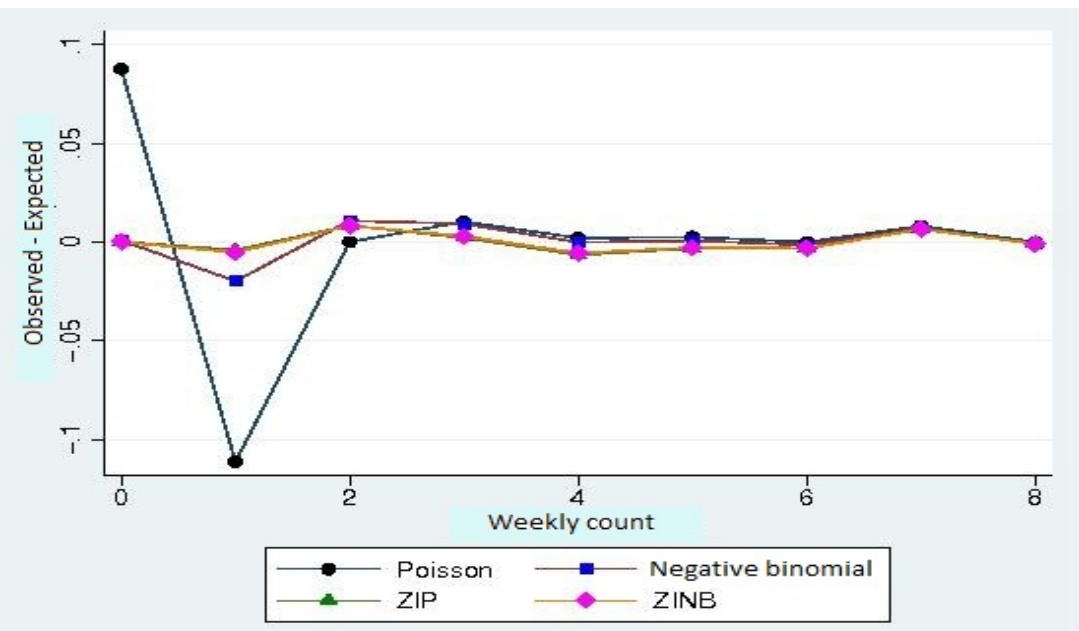

Fig. 4 Difference between the proportion observed and the mean likelihoods from the four models fitted for leisure-time physical activity performance (rigorous level). Botucatu, 2003.

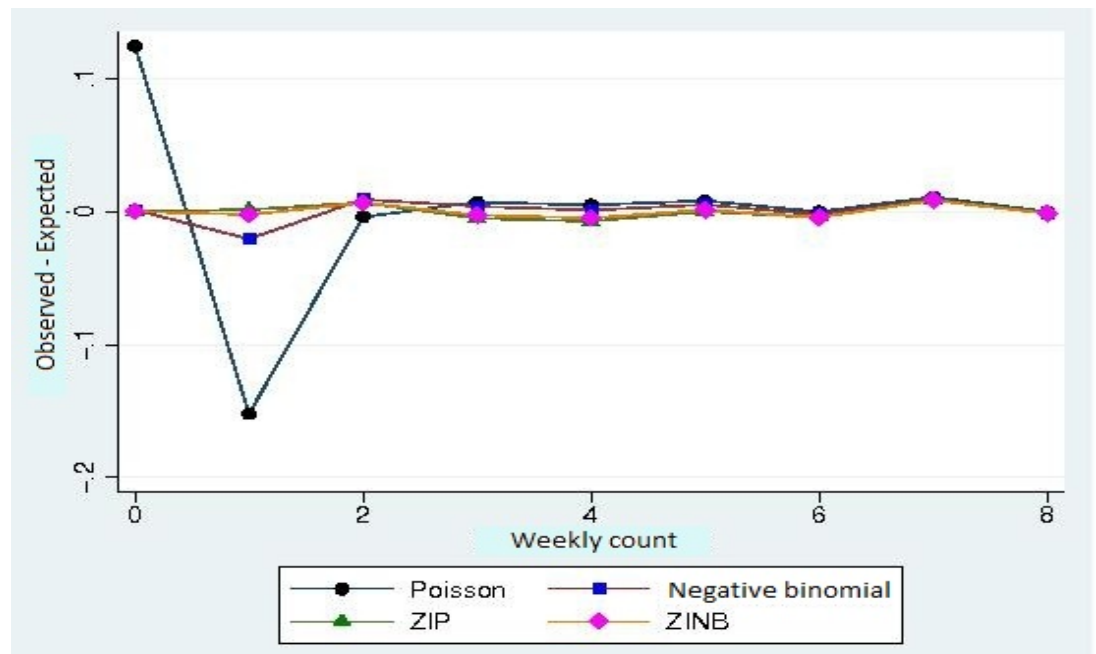

Fig. 5 Difference between the proportion observed and the mean likelihoods from the four models fitted for leisure-time physical activity performance (moderate level). Botucatu, 2003.

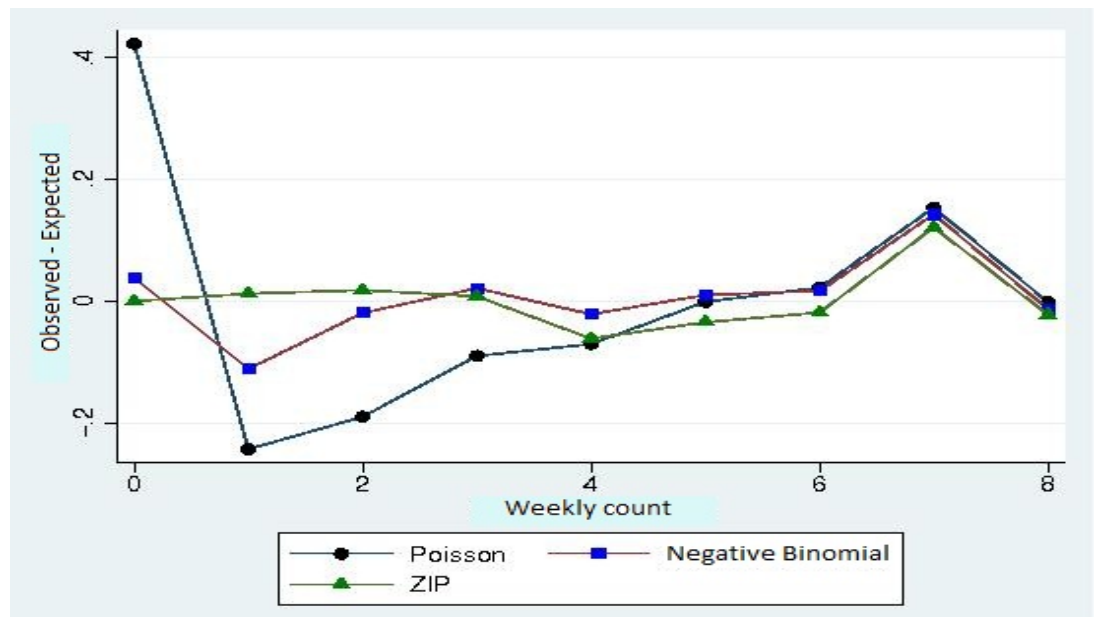

Fig. 6 Difference between the proportion observed and the mean likelihoods from the four models fitted for leisure-time physical activity performance (light level). Botucatu, 2003. 
such as walking. Satisfaction about life and the fact of not having a companion showed to be decisive factors for not performing recreation, sports or leisure activities. Additionally, poor schooling showed to be decisive for not performing physical activities both at home and during leisure. The study also confirmed two daily pieces of evidence: as age advances, the performance of simple activities at home decreases, and that older individuals who still participate in the work market are more willing to perform household tasks.

The information collected in this study is valuable in order to propose physical activity programs for older individuals by means of public policies targeted at this age range, thus promoting healthy and quality ageing essentially by preventing chronic and degenerative diseases.

\section{References}

[1] B. Frye, S. Scheinthal, T. Kemarskaya, R. Pruchno, Tai chi and low impact exercise: Effects on the physical functioning and psychological well-being of older people, Journal of Applied Gerontology 26 (5) (2007) 433-453.

[2] I.M. Lee, R.S. Paffenbarger, Associations of light, moderate, and vigorous intensity physical activity with longetivy, The Harvard Alumni Health Study, Am. J. Epidemiol. 2 (2000) 108-114.

[3] H.D. Sesso, R.S. Pafferbarger, L.M. Lee, Physical activity and coronary heart disease in men: The Harvard Alumni Study, Circulation 102 (2000) 178-185.

[4] M.E. Nelson, W.L. Rejaski, S.N. Blair, P.M. Duncan, J.O. Judge, A.C. King, et al., Physical activity and public health in older adults: recommendation from the American College of Sports Medicine and the American Heart Association, Medicine \& Science in Sports \& Exercise 39 (8) 2007 1435-35.

[5] S.M. Matsudo, V.K.R. Matsudo, T.L.B. Neto, Atividade física e envelhecimento: Aspectos epidemiológicos, Brazilian Journal of Medicine and Sports 1 (2001) 20-26.

[6] P. Ainslie, T. Reilly, K. Westerterp, Estimating human energy expenditure: A review of techniques with particular reference to doubly labelled water, Medicine \& Science in Sports \& Exercise 33 (9) (2003) 683-698.

[7] C.L. Craig, A.L. Marshall, M. Sjöström, A.E. Bauman,
M.L. Booth, B.E. Ainsworth, et al., Internacional physical activity questionaire: twelve country reliability and validity, Medicine \& Science in Sports \& Exercise 35 (2003) 1381-1395.

[8] T.R.B. Benedetti, G.Z. Mazo, M.V.G. Barros, Application of the International Physical Activity Questionnaire (IPAQ) for evaluation of elderly women: concurrent validity and test-retest reproducibility, Brazilian Journa of Science and Movement 12 (1) (2004) 25-34.

[9] T.R.B. Benedetti, P.C. Antunes, C.R. Rodriguez-Anez, G.Z. Mazo, E.L. Petroski, Reproducibility and validity of the International Physical Activity Questionnaire (IPAQ) in older men, Brazilian Journal of Medicine and Sport 13 (1) (2007) 11-16.

[10] D.J. Slymen, G.X. Ayala, E.M. Arredondo, J.P. Elder, A demonstration of modeling count data with an application to physical activity, Epidemiologic Perspectives \& Innovations 3 (2006) 1-9.

[11] R. Cordeiro, M. Sakake, A.P.G. Clemente, C.S. Diniz, M.R. Donalisio, Underreporting of non-fatal work-related injuries in Brazil, Rev. Saúde Pública 39 (2005) 254-260.

[12] J.S. Long, J. Freese, Predicted probabilities for count models, The Stata Journal 1 (1) (2001) 51-57.

[13] Q.H. Wuong, Likelihood ratio tests for model selection and non-nested hypotheses, Econometrica 52 (2) (1989) 307-333.

[14] J. Kuha, AIC e BIC: Comparisons of assumptions and performance, Sociological Methods Research 33 (2) (2004) 188-229.

[15] L.M.C. Rolim, Level of physical activity associated to the lifestyle and satisfaction with life of the older poplation of the Botucatu city, São Paulo, Brazil, Msc. Thesis, 2008.

[16] M.P. Zaitune, M.B.A. Barros, C.L.G. César, L. Carandina, M. Goldbaum, Variables associated to the sedentary leasure time in the elderly in Campinas, São Paulo State, Brasil, Reports in Public Health 23 (6) (2007) 1329-1338.

[17] F.V. Siqueira, L.A. Facchini, R.X. Piccini, Physical actitivy in young adults and the elderly in areas covered by primary health care units in municipalities in the South and Northeast of Brasil, Reports in Public Health 24 (1) (2008) 39-54.

[18] C.A. Monteiro, W.L. Conde, S.M. Matsudo, V.K.R. Matsudo, I.M. Bonseñor, P.A. Lutufo, A descriptive epidemiology of leisure-time of physical activity in Brazil, 1996-1997, Pan American Journal of Public Health 14 (2003) 246-254. 